\title{
An assessment of couple stress theories
}

\author{
Ali R. Hadjesfandiari, Gary F. Dargush \\ Department of Mechanical and Aerospace Engineering \\ University at Buffalo, The State University of New York, Buffalo, NY 14260 USA \\ ah@buffalo.edu,gdargush@buffalo.edu
}

November 7, 2018

\begin{abstract}
In this paper, we examine the mathematical and physical consistencies of the three primary couple stress theories: original Mindlin-Tiersten-Koiter couple stress theory (MTK-CST), modified couple stress theory (M-CST) and consistent couple stress theory (C-CST). As has been known for many years, MTK-CST suffers from some fundamental inconsistencies, such as the indeterminacy of the couple-stress tensor. Therefore, despite the fact that MTK-CST has a fundamental position in the evolution of size-dependent continuum mechanics, it is not a reliable theory within continuum mechanics, for example, in developing new size-dependent multi-physics formulations. We also observe that M-CST not only inherits all inconsistences from the original MTK-CST, but also suffers from new additional inconsistencies, such as the introduction of a new non-physical governing equation. These inconsistencies refute the claim of those who state that the couple-stress tensor may be chosen symmetric. Therefore, the apparent success of MTK-CST and M-CST in describing a size-effect for some problems, such as two-dimensional plate and beam bending, is not enough to justify these theories as suitable for general cases. In fact, the symmetric couple-stresses in M-CST create torsional or anticlastic deformation, not bending. On the other hand, C-CST, with a skew-symmetric couple-stress tensor, is the consistent continuum mechanics suitable for solving different size-dependent solid, fluid and multi-physics problems.
\end{abstract}

Keywords: Couple stress theory; Size-dependent mechanics; Indeterminacies; Curvature tensor; Torsion tensor 


\section{Introduction}

More than a half-century ago, Mindlin and Tiersten (1962) and Koiter (1964) developed an initial incomplete version of couple stress theory (MTK-CST), which uses the four foundational continuum mechanical quantities (i.e., force, displacement, couple, rotation). However, this theory suffers from some fundamental inconsistencies, such as the indeterminacy of the couple-stress tensor (Eringen, 1968), and the appearance of the normal component of the couple-traction vector on boundary surfaces. Therefore, the first step in using any couple stress theory should be resolving the criticism of Eringen (1968) about the indeterminacy of couple-stress tensor in MTKCST. For linear isotropic elastic materials, this theory requires two couple-stress material parameters, which also does not seem attractive from a practical point of view.

Without answering the criticism of Eringen (1968) about the indeterminacy of couple-stress tensor in MTK-CST, Yang et al. (2002) violated the fundamental rules of mechanics to reduce the number of couple-stress material parameters in this theory for linear isotropic elastic material from two coefficients to only one. In this awkward development, they introduced an extra artificial equilibrium equation for the moment of couples that has no physical reality, but apparently makes the couple-stress tensor symmetric. However, in this still indeterminate theory, called modified couple stress theory (M-CST), the symmetric couple-stress tensor has a torsional character, which results in torsional and anticlastic deformation, rather than bending. Consequently, this theory predicts deformations, which contradict common sense and do not agree with physical experiments. Furthermore, as in MTK-CST, the boundary condition specification in M-CST is often problematic.

In a more recent development, Hadjesfandiari and Dargush (2011) resolved the inconsistencies of MTK-CST by discovering the subtle skew-symmetric character of the couple-stress tensor. Interestingly, this result shows that the couple-stress is a true vector. Therefore, in this theory, called consistent couple stress theory (C-CST), the couple-stress tensor is determinate and creates bending deformation. Remarkably, for linear isotropic elastic material, this theory requires only one couple-stress material parameter. Consequently, C-CST resolves the quest for the consistent size-dependent continuum mechanics by answering the criticism of Eringen, and provides a fundamental basis for the development of size-dependent material response. 
The remainder of the paper is organized as follows. In Section 2, we provide an overview of the original MTK-CST, which outlines its inconsistencies. In Section 3, we examine M-CST, which does not answer the criticism of Eringen about the indeterminacy of the couple-stress tensor in MTK-CST. We demonstrate that this torsional-based inconsistent couple stress theory has created even more confusion. In Section 4, we briefly present C-CST, which resolves all inconsistencies in MTK-CST. In Section 5, we discuss several physical and mathematical inconsistencies of the original MTK-CST and M-CST. Finally, we offer some conclusions in Section 6.

\section{Original couple stress theory}

The original Mindlin-Tiersten-Koiter couple stress theory (MTK-CST) (Mindlin and Tiersten, 1962; Koiter, 1964) is a fundamental pillar in the development of size-dependent continuum mechanics. This theory is based on considering the motion of infinitesimal elements of matter at each point of the continuum. This means that MTK-CST is an extension of rigid body mechanics, which then is recovered in the absence of deformation (Hadjesfandiari and Dargush, 2015b). Therefore, in this theory, the displacements $u_{i}$ and rotations $\omega_{i}$ provide the primary degrees of freedom of infinitesimal elements of the continuum at each point. However, the continuity of matter within the continuum description restrains the rotation field $\omega_{i}$ to equal one-half the curl of the displacement field $u_{i}$

$$
\omega_{i}=\frac{1}{2} \varepsilon_{i j k} u_{k, j} \quad \boldsymbol{\omega}=\frac{1}{2} \nabla \times \mathbf{u}
$$

where $\varepsilon_{i j k}$ is the Levi-Civita alternating symbol.

For the continuum, the internal stresses are represented by force-stress $\sigma_{i j}$ and couple-stress $\mu_{i j}$ tensors (Voigt, 1887; Cosserat and Cosserat, 1909). Therefore, the force-traction vector $t_{i}^{(n)}$ and couple-traction vector $m_{i}^{(n)}$ through a surface element $d S$ in the volume with outward directed unit normal $n_{i}$ are given, respectively, as

$$
t_{i}^{(n)}=\sigma_{j i} n_{j}
$$




$$
m_{i}^{(n)}=\mu_{j i} n_{j}
$$

The fundamental governing equations in MTK-CST are based on the force and moment equations for system of particles (Hadjesfandiari and Dargush, 2018a)

$$
\begin{array}{cc}
\sum \mathbf{F}^{e x t}=\sum m \mathbf{a} & \sum F_{i}^{e x t}=\sum m a_{i} \\
\sum \mathbf{M}^{e x t}=\sum \mathbf{r} \times m \mathbf{a} & \sum M_{i}^{e x t}=\varepsilon_{i j k} \sum x_{j} m a_{k}
\end{array}
$$

where $\sum F_{i}$ and the moment $\sum M_{i}$ are the resultant of external forces and external moments, respectively. We notice that these equations are the result of Newton's second and third laws for the system of particles. Therefore, by considering an arbitrary part of the material continuum occupying a volume $V_{a}$ enclosed by boundary surface $S_{a}$, the force and moment equations for this part of the body become

$$
\begin{gathered}
\int_{S_{a}} \mathbf{t}^{(n)} d S+\int_{V_{a}} \mathbf{f} d V=\int_{V_{a}} \rho \mathbf{a} d V \\
\int_{S_{a}}\left[\mathbf{r} \times \mathbf{t}^{(n)}+\mathbf{m}^{(n)}\right] d S+\int_{V_{a}} \mathbf{r} \times \mathbf{f} d V=\int_{V_{a}} \mathbf{r} \times \rho \mathbf{a} d V
\end{gathered}
$$

or in terms of components

$$
\begin{gathered}
\int_{S_{a}} t_{i}^{(n)} d S+\int_{V_{a}} f_{i} d V=\int_{V_{a}} \rho a_{i} d V \\
\int_{S_{a}}\left[\varepsilon_{i j k} x_{j} t_{k}^{(n)}+m_{i}^{(n)}\right] d S+\int_{V_{a}} \varepsilon_{i j k} x_{j} f_{k} d V=\int_{V_{a}} \varepsilon_{i j k} x_{j} \rho a_{k} d V
\end{gathered}
$$

where $f_{i}$ is the specified body-force density, $\rho$ is the mass density, and $a_{i}$ is the acceleration vector. For small deformation theory, we can use the approximation $a_{i}=\frac{\partial^{2} u_{i}}{\partial t^{2}}=\ddot{u}_{i}$.

The force and moment equations (6) and (7) are the only governing equations for the material continuum, in which the effect of internal forces in the volume $V_{a}$ are cancelled based on Newton's third law of action and reaction (Hadjesfandiari and Dargush, 2018a). It is impossible to write any new independent governing equation, in which internal forces disappear. Although defining 
alternative and independent moments of forces and momentum is possible, they do not have any physical significance, because the effect of internal forces does not disappear in the corresponding equations (Hadjesfandiari and Dargush, 2018a).

By using the relations (2) and (3) in the governing equations (8) and (9), along with the divergence theorem, and noticing the arbitrariness of volume $V_{a}$, we finally obtain the differential form of the equilibrium equations, for an infinitesimal element of matter, as (Hadjesfandiari and Dargush, 2011, 2015b):

$$
\begin{array}{ll}
u_{i} \leftrightarrow 3 \text { force equations } & \sigma_{j i, j}+f_{i}=\rho \ddot{u}_{i} \\
\omega_{i} \leftrightarrow 3 \text { moment equations } & \mu_{j i, j}+\varepsilon_{i j k} \sigma_{j k}=0
\end{array}
$$

The three force equations (10) correspond to the translational motions $u_{i}$ and the three moment equations (11) correspond to the rotational motions $\omega_{i}$ of the infinitesimal element of matter.

Mindlin and Tiersten (1962) and Koiter (1964) have shown that the displacement field $u_{i}$ prescribed on a smooth part of the boundary $S$ specifies the normal component of the rotation $\omega^{(n n)}=\omega_{i} n_{i}$. Accordingly, they have demonstrated that material in a consistent couple stress theory does not support independent distributions of normal surface couple (or twisting) traction $m^{(n n)}=m_{i}^{(n)} n_{i}$. This means

$$
m^{(n n)}=m_{i}^{(n)} n_{i}=\mu_{j i} n_{i} n_{j}=0
$$

Consequently, Mindlin and Tiersten (1962) and Koiter (1964) correctly established that five geometrical and five mechanical boundary conditions can be specified on a smooth surface. However, they did not realize the fundamental implication of (12) on the character of the couplestress tensor and its energetically conjugate curvature tensor. As a result, in their development the rotation $k_{i j}=\omega_{j, i}$, called the bend-twist tensor (deWit, 1970), is considered as the curvature tensor measure of deformation. However, the deviatoric character of $k_{i j}$ makes the couple-stress tensor indeterminate. 
In the original MTK-CST, Mindlin and Tiersten have also considered the existence of the body couple in the formulation. However, this results in the disturbing appearance of the body couple in the relation for the force-stress tensor (Hadjesfandiari and Dargush, 2011). Interestingly, Mindlin and Tiersten (1962) have transformed the body couple into an equivalent body force in the volume and a force-traction vector on the bounding surface. However, they did not realize the fundamental meaning of this transformation that the body couple is not distinguishable from the body force (Hadjesfandiari and Dargush, 2011).

For linear isotropic elastic material, the constitutive relations in this theory are

$$
\begin{gathered}
\sigma_{(i j)}=\lambda e_{k k} \delta_{i j}+2 \mu e_{i j} \\
\mu_{i j}=Q \delta_{i j}+4 \eta k_{i j}+4 \eta^{\prime} k_{j i} \\
=Q \delta_{i j}+4 \eta \omega_{j, i}+4 \eta^{\prime} \omega_{i, j}
\end{gathered}
$$

where $Q \delta_{i j}$ is the indeterminate spherical part of the couple-stress tensor, and $\lambda$ and $\mu$ are the Lamé coefficients for isotropic media. The parameters $\eta$ and $\eta^{\prime}$ are the couple-stress material coefficients for isotropic media. However, the appearance of two couple-stress material parameters is a side effect of the indeterminacy of the couple-stress tensor in this theory, which is also troublesome in practice.

Consequently, the original MTK-CST suffers from some serious inconsistencies and difficulties with the underlying formulations, which may be summarized as follows:

1. The inconsistency in boundary conditions, since the normal component of the coupletraction vector $m^{(n n)}=m_{i} n_{i}$ appears in the formulation violating the condition (12);

2. The appearance of the indeterminate component $Q \delta_{i j}$ as the spherical part of the couplestress tensor, and thus, in the skew-symmetric part of the force-stress tensor;

3. The disturbing appearance of the body couple in the relation for the force-stress tensor; 
4. The appearance of two couple-stress elastic coefficients $\eta$ and $\eta^{\prime}$ for linear elastic isotropic material, although only one of these elastic coefficients, $\eta$, appears in the final governing equations, when written in terms of displacements.

The appearance of an arbitrary spherical couple-stress component $Q \delta_{i j}$ in the couple-stress tensor is the result of the deviatoric or trace free $\omega_{i, i}=0$ character of the bend-twist tensor $k_{i j}=\omega_{j, i}$ in this theory. This clearly shows that the bend-twist tensor $k_{i j}=\omega_{j, i}$ is not a proper measure of deformation in couple stress theory. The disturbing non-physical character of the spherical component $Q \delta_{i j}$ is that it does not create any deformation in the body, which means its effect is equivalent to a zero loading condition. Although this spherical part $Q \delta_{i j}$ also affects the state of couple-stress and force-stress tensors through boundary conditions, it disappears in the final governing equations when written in terms of displacements. This spherical part of the couplestress tensor is troublesome in most cases, especially those with torsional deformation, and thus cannot be simply ignored. Koiter (1964) has claimed that without any physical loss in generality the indeterminate spherical part may be taken zero. However, this is not the case.

Eringen (1968) realized this inconsistency as a major mathematical problem in the original MTKCST, which he afterwards called indeterminate couple stress theory. This means before using couple stress theory, we must answer the criticism of Eringen (1968) concerning the indeterminacy of the couple-stress tensor in MTK-CST. However, Mindlin and others were not able to resolve this trouble in the framework of MTK-CST. Interestingly, Mindlin never used MTK-CST to solve any problem or to develop any formulation after Eringen's criticism. As a result, Mindlin and others turned to alternative theories, such as micropolar theories (Eringen (1968) and strain gradient theories (Mindlin, 1965; Mindlin and Eshel, 1968). However, it is very strange to see that after half a century some researchers claim that there is no problem in an indeterminate couple stress theory (Neff et al., 2016; Ghiba et al., 2016; Münch et al., 2015). These authors do not recognize that MTK-CST boundary value problems are ill-posed, which obviously shows that these authors have some "fundamental misunderstandings in the indeterminate couple stress theory" (Hadjesfandiari and Dargush, 2016a). We realize now that not resolving the indeterminacy 
issue has cost mechanics a great deal and confused its progress by creating many non-physical concepts and theories, such as strain gradient theories (Hadjesfandiari and Dargush, 2018b).

The appearance of the normal component of the couple-traction vector $m^{(n n)}=m_{i} n_{i}$ in MTK-CST is also very disturbing. To resolve this problem, Koiter (1964) proposed that a distribution of normal surface twisting couple-traction $m^{(n n)}$ on the actual surface $S$ be replaced by an equivalent shear stress distribution and a line force system. However, the fundamental difficulty with this boundary condition and its impact on MTK-CST was not appreciated at the time. Mindlin and Tiersten (1962) and Koiter (1964) did not realize that the boundary condition $m^{(n n)}=0$ is a fundamental character of couple stress theory, which must be satisfied systematically once and for all (Hadjesfandiari and Dargush, 2011, 2015a,b). Unfortunately, the introduction of the approximate reduced boundary condition method from structural mechanics by Koiter (1964) has caused the majority of researchers to forget about the fundamental boundary condition $m^{(n n)}=0$.

Consequently, from a practical point of view, it is generally impossible to satisfy all boundary conditions correctly in many problems using MTK-CST. For example, there is no consistent solution for pure torsion of a circular bar in this theory (Koiter, 1964), and the approximate inconsistent solution for pure torsion in MTK-CST may predict significant size effect, which does not agree with experiments (Lu and Song, 2011; Song and Lu, 2015; Hadjesfandiari and Dargush, 2016b). In addition, MTK-CST cannot describe the pure bending of a plate properly (Hoffman, 1964; Ellis and Smith, 1967; Hadjesfandiari et al., 2016). The inconsistency of MTK-CST also can be seen in the anti-plane deformation (Radi, 2008; Mishuris et al., 2012; Morini et al., 2014; Gavardinas et al., 2017; Zisis, 2018), where there are no consistent exact solutions.

It is very disappointing to see that proponents of MTK-CST do not realize the severity of Eringen's criticism about the indeterminacy, and simply ignore $Q \delta_{i j}$ without any reasoning. A couple stress theory with a deviatoric couple-stress tensor is not physically acceptable (Hadjesfandiari and Dargush, 2015b). We should realize that in MTK-CST with deviatoric couple-stresses, uniaxial torsional deformation with a normal couple-stress is impossible, which contradicts the physical reality. Based on physical grounds, if we can exert torsional couple-stresses $\mu_{11}, \mu_{22}$ and $\mu_{33}$ on 
some element of the matter, these three components must be independent of each other. This physical fact contradicts the mathematical deviatoric condition, $\mu_{i i}=\mu_{11}+\mu_{22}+\mu_{33}=0$. Therefore, the first step in using MTK-CST must be resolving the aforementioned difficulties, especially answering the criticism of Eringen (1968) about the indeterminacy of the couple-stress tensor.

Stokes (1966) brought the original MTK-CST into fluid mechanics to model the size-dependency effect in fluids. The constitutive relations in this theory depend on three material parameter constants: the classical viscosity $\hat{\mu}$ and two additional couple-stress viscous parameters $\hat{\eta}$ and $\hat{\eta}^{\prime}$ (Stokes, 1966). Obviously, this theory suffers from the same serious inconsistencies and difficulties present in the underlying formulations for solids. In translating the theory to fluids, Stokes $(1966,1984)$ did not incorporate the known fundamental character of boundary conditions, as defined earlier by Mindlin, Tiersten (1962) and Koiter (1964), which allows only five geometrical (essential) and mechanical (natural) boundary conditions to be specified on a smooth surface in three-dimensional space, not six. As a result, most followers of the Stokes couple stress fluid theory do not appreciate the basic shortcomings of this theory, such as the indeterminacy issue and ill-posed boundary conditions.

Stokes $(1966,1984)$ also suggests without substantiation that the spherical part of the couple-stress tensor either can come through from boundary conditions or may be assumed to be zero without any loss of generality. This suggestion works for two-dimensional flows, where the Stokes formulation may give valid velocity solutions independent of the couple-stress parameter $\hat{\eta}^{\prime}$ (e.g, Stokes, 1971; Shivakumara et al., 2014; Turkyilmazoglu, 2014; Shankar et al., 2014; Shankar et al., 2016; Srinivasacharya et al., 2016; Mahabaleshwar et al., 2017; Tripathi et al., 2017; Nandal and Mahajan, 2018; Layek and Pati, 2018). However, this does not make Stokes' couple stress fluid mechanics development a viable theory to replace the Navier-Stokes fluid mechanics for solving all problems. In particular, the suggestion by Stokes is not true for general threedimensional cases, especially when there are torsional deformations or mixed boundary conditions (Hadjesfandiari et al., 2015). For these cases, the velocity solution and the couple-stress distribution depend on the additional couple-stress parameter $\hat{\eta}^{\prime}$. This can be seen for Poiseuille 
flow through circular pipes (Stokes, 1966, 1984), where the velocity profile and stresses depend on both viscous parameters $\hat{\eta}$ and $\hat{\eta}^{\prime}$. It turns out in some three-dimensional cases with velocity boundary conditions the results for velocities may look fine (Sunil et al., 2011, 2013; Hayat et al., 2015; Hayat et al.,2017). However, the couple-stress distribution inside the fluid depends on $\hat{\eta}^{\prime}$. The proponents of Stokes' couple stress fluid mechanics should notice that the peculiar character of this theory in producing valid velocity solutions for some cases in linear isotropic viscous couple stress fluids does not lead to generality. The extension of the Stokes couple stress theory to anisotropic or non-Newtonian fluids would be especially problematic. Although the Stokes' theory requires two couple-stress viscous parameters $\hat{\eta}$ and $\hat{\eta}^{\prime}$ for linear isotropic fluids, it is not known why in very recent work Laouadi et al. (2018) incorrectly state that this theory depends on one couple-stress viscous parameter $\hat{\eta}$. As mentioned, the dependence of the velocity profile and stresses on both viscous parameters $\hat{\eta}$ and $\hat{\eta}^{\prime}$ is obviously seen for Poiseuille flow through circular pipes (Stokes, 1966, 1984).

\section{Modified couple stress theory}

By some manipulation of the differential form of the moment equation (11), we can obtain different forms of the equations, which are not independent of the original moment equation (11). For example, by multiplying the moment equation (11) by $\varepsilon_{i j k} x_{j}$, we obtain

$$
\varepsilon_{i j k} x_{j} \mu_{l k, l}+\varepsilon_{i j k} x_{j} \varepsilon_{k p q} \sigma_{p q}=0
$$

This is the moment of the moment equation (11) about the origin, which can be written as

$$
\left(\varepsilon_{i j k} x_{j} \mu_{l k}\right)_{, l}-\varepsilon_{i j k} \delta_{j l} \mu_{l k}+\varepsilon_{k i j} x_{j} \varepsilon_{k p q} \sigma_{p q}=0
$$

Now by integrating in the volume $V_{a}$, using (3) and applying the divergence theorem, we obtain

$$
\int_{S_{a}} \varepsilon_{i j k} x_{j} m_{k}^{(n)} d S-\int_{V_{a}}\left(\varepsilon_{i j k} \mu_{j k}-\varepsilon_{i j k} x_{j} \varepsilon_{k p q} \sigma_{p q}\right) d V=0
$$

Interestingly, this can also be written in the vectorial form as

$$
\int_{S_{a}} \mathbf{r} \times \mathbf{m}^{(n)} d S-\int_{V_{a}}[\boldsymbol{\epsilon}: \boldsymbol{\mu}-\mathbf{r} \times(\boldsymbol{\epsilon}: \boldsymbol{\sigma})] d V=0
$$


We emphasize that this equation is not a new governing equation of motion independent of the moment equation (7).

In a misleading paper, Yang et al. (2002) concentrated only on reducing the number of couplestress material parameters for a linear isotropic elastic material from two coefficients $\eta$ and $\eta^{\prime}$ to only one $\eta$, without realizing that the indeterminacy of the couple-stress tensor is the main reason for the appearance of two coefficients. For this purpose, Yang et al. (2002) introduced an extra artificial equilibrium equation for the moment of couples

$$
\int_{S} \mathbf{r} \times \mathbf{m}^{(n)} d S+\int_{V} \mathbf{r} \times(\epsilon: \boldsymbol{\sigma}) d V=0
$$

in addition to the two conventional vectorial force and moment equations (6) and (7). However, we notice that this equation has no physical meaning. It also does not have a discrete analog similar to the force and moment governing equations (4) and (5) for a system of particles. Examining the derivation of the artificial equation (19) shows that Yang et al. (2002) have had difficulties in using classical mechanics rules (Hadjesfandiari and Dargush, 2015a) and it has been noted that this equation is not derived by any principle of classical mechanics (Lazopoulos, 2009; Hadjesfandiari and Dargush, 2015a; Münch et al., 2015). Furthermore, this new equation (19) has no physical explanation and does not correspond to any degrees of freedom of the rigid-like motion of infinitesimal elements of matter. Surprisingly, we realize that in a mechanics with the additional equation (19), the rigid body portion of motion of infinitesimal elements of matter at each point of the continuum is described by nine degrees of freedom, three displacements $u_{i}$, three rotations $\omega_{i}$ and three unknown motions $\xi_{i}$, where

$$
\begin{gathered}
u_{i} \leftrightarrow 3 \text { force equations } \\
\omega_{i} \leftrightarrow 3 \text { moment equations } \\
\xi_{i} \leftrightarrow 3 \text { moment of couple equations }
\end{gathered}
$$

Therefore, the force and moment equations (6) and (7) describe the translational and rotational motion of the infinitesimal elements of matter, but the unsubstantiated moment of couple equation 
(19) describes a non-physical motion corresponding to the artificial unknown vector degrees of freedom $\xi_{i}$.

Application of the unsubstantiated non-physical equilibrium equation (19) in (18), leads to a symmetric couple-stress tensor

$$
\mu_{j i}=\mu_{i j}
$$

As a result, in this modified couple stress theory (M-CST), the symmetric part $\chi_{i j}$ of the bendtwist tensor $k_{i j}=\omega_{j, i}$, which can be written

$$
\chi_{i j}=\omega_{(i, j)}=\frac{1}{2}\left(\omega_{i, j}+\omega_{j, i}\right)
$$

is the curvature tensor measure of deformation. However, we notice that $\chi_{i j}$ is a torsion tensor, not a curvature tensor. In principal coordinates $x_{1}^{\prime} x_{2}^{\prime} x_{3}^{\prime}$, this tensor becomes diagonal representing pure torsional deformations, where

$$
\left[\chi_{i j}^{\prime}\right]=\left[\begin{array}{ccc}
\chi_{11}^{\prime} & 0 & 0 \\
0 & \chi_{22}^{\prime} & 0 \\
0 & 0 & \chi_{33}^{\prime}
\end{array}\right]=\left[\begin{array}{ccc}
\omega_{1,1}^{\prime} & 0 & 0 \\
0 & \omega_{2,2}^{\prime} & 0 \\
0 & 0 & \omega_{3,3}^{\prime}
\end{array}\right]
$$

This means that the couple-stresses in M-CST create torsional deformation, rather than bending. As a result, the normal couple-stress components on plane element surfaces create torsion, and tangential components deform these plane elements into anticlastic surfaces.

For linear isotropic elastic material in M-CST, the constitutive relations apparently are

$$
\begin{aligned}
& \sigma_{(i j)}=\lambda e_{k k} \delta_{i j}+2 \mu e_{i j} \\
& \mu_{i j}=Q \delta_{i j}+8 \eta \chi_{i j} \\
& =Q \delta_{i j}+4 \eta\left(\omega_{i, j}+\omega_{j, i}\right)
\end{aligned}
$$

Since the torsion tensor is deviatoric or trace free, that is $\chi_{i i}=\omega_{i, i}=0$, the symmetric couplestress tensor $\mu_{i j}$ in this theory is still indeterminate. 
Similar to the original MTK-CST, Yang et al. (2002) have also considered the existence of the body couple in their formulation, which causes the disturbing appearance of the body couple in the relation for the force-stress tensor. Consequently, M-CST not only inherits all inconsistences from original MTK-CST, but also suffers from the unsubstantiated additional artificial equilibrium of moment of couples (19) in the set of fundamental governing equations. Instead of realizing that the peculiar appearance of the additional couple-stress elastic coefficient $\eta^{\prime}$ for linear elastic isotropic materials is the result of the indeterminacy of couple-stress tensor and boundary conditions, Yang et al. (2002) have violated the fundamental rules of physics and mechanics with disastrous consequences.

It is really surprising to see that numerous researchers have fallen for the unsubstantiated equation (19) and have tried to even generalize it further in continuum mechanics (Surana et al., 2017; Surana et al. 2015a,b). However, these authors do not realize that the only possible independent governing equations of motion are the force and moment equations (6) and (7), in which the internal forces in any volume of matter as a system cancel based on Newton's third law of action and reaction. It is impossible to write any new independent governing equation. It should be noticed that although defining higher moments of forces and momentum is possible, they do not have any physical significance (Hadjesfandiari and Dargush, 2018a). This is because the effect of internal forces does not disappear for any higher moment equations, except those developed from (7). It is only for the first moment equation (7) that the moment of internal forces cancel based on Newton's third law of action and reaction. This is the reason why the force and moment equations (6) and (7) are considered as fundamental laws in mechanics.

If the moment of couple equilibrium equations (19) were true, all physical science from classical rigid bodies to atomic, nuclear and particle physics would need to be changed. However, this nonphysical equation has been just invented to make the couple-stress tensor symmetric without any application anywhere else. This is the reason why Newton, Euler, Lagrange and Hamilton did not discover this apparently important, but completely non-physical equation in the first place. 
Although Neff and his colleagues (Münch et al., 2015) agree that the moment of couple equation used by Yang et al. (2002) is not physical, they still suggest the couple-stress tensor can be chosen symmetric. These authors do not realize that a symmetric couple stress theory still suffers from the same inconsistencies and difficulties with the underlying formulation as MTK-CST. As in the original MTK-CST, the spherical part of the couple-stress tensor $Q \delta_{i j}$ also remains indeterminate in this theory and cannot be determined in a consistent systematic way. It is surprising to see that Neff and his colleagues and the other proponents of M-CST theory simply ignore $Q \delta_{i j}$ without any reasoning and assume the couple-stress tensor is deviatoric. Furthermore, the symmetric couple-stress tensor in this theory has a torsional character, which results in torsional and anticlastic deformation, not bending.

It is generally impossible to satisfy all boundary conditions correctly in the solution of most problems in M-CST. This can be observed for the pure torsion of a circular bar, where it is impossible to obtain an exact M-CST solution. Furthermore, the apparent approximate pure torsion solution predicts a significant size-effect, which contradicts recent experiments for pure torsion of micro-diameter copper wires (Lu and Song, 2011; Song and Lu, 2015; Hadjesfandiari and Dargush, 2016b). In addition, M-CST cannot describe the pure bending of a plate properly (Hadjesfandiari et al., 2016). The normal force-stresses create bending deformation as in the classical theory, whereas the couple-stresses create torsional deformation. Surprisingly, M-CST predicts no couple-stresses and no size-effect for the pure bending of the plate into a spherical shell. These characteristics also make M-CST of questionable value to serve as a basis for sizedependent structural models, such as beams, plates and shells. Perhaps, this character of M-CST has not been fully understood, but in any case, it is unfortunate that this theory has been used so extensively in structural mechanics, especially in one dimensional beam bending problems (e.g., Park and Gao, 2006; Kong et al., 2008; Ma et al., 2008; Asghari et al., 2010, 2011; Reddy, 2011; Li et al., 2014; Chen et al., 2015; Gao, 2015; Karttunen e al., 2016; Goncalves et al., 2017; Goncalves and Romanoff, 2018). 


\section{Consistent couple stress theory (C-CST)}

Recently, Hadjesfandiari and Dargush (2011, 2015b) and Hadjesfandiari et al. (2015) have developed consistent couple stress theory (C-CST), which resolves all inconsistencies in the original MTK-CST. The main achievement of this development is discovering the subtle skewsymmetric character of the couple-stress tensor

$$
\mu_{j i}=-\mu_{i j}
$$

The fundamental step in this development is satisfying the requirement (12) that the normal component of the couple-traction vector must vanish on the boundary surface in a systematic way, i.e. $m^{(n n)}=\mu_{j i} n_{j} n_{i}=0$. Hadjesfandiari and Dargush (2011) have also shown that the body couple is not distinguishable from the body force. Therefore, in a proper couple stress theory the concept of body couple is redundant, and we must only consider body forces.

In C-CST, the negative of the skew-symmetric part of the bend-twist tensor, $k_{i j}=\omega_{j, i}$, that is,

$$
\kappa_{i j}=\omega_{[i, j]}=\frac{1}{2}\left(\omega_{i, j}-\omega_{j, i}\right)
$$

is the curvature tensor measure of deformation. This tensor, which is called the mean curvature tensor, is energetically conjugate to the skew-symmetric couple-stress tensor $\mu_{i j}$. We notice that the skew-symmetric couple-stresses create synclastic or ellipsoidal cap-like deformation with positive Gaussian curvature for surface elements of the continuum.

Interestingly, the skew-symmetric tensors $\mu_{i j}$ and $\kappa_{i j}$ can be represented by their dual true couplestress vector $\mu_{i}$ and true mean curvature vector $\kappa_{i}$ (Hadjesfandiari and Dargush, 2011), where

$$
\begin{gathered}
\mu_{i}=\frac{1}{2} \varepsilon_{i j k} \mu_{k j} \\
\kappa_{i}=\frac{1}{2} \varepsilon_{i j k} \kappa_{k j}
\end{gathered}
$$


Therefore, consistent skew-symmetric couple stress theory may be called the vector couple stress theory. It is amazing to notice that the skew-symmetric character of the couple-stress tensor immediately resolves the indeterminacy problem by establishing that there is no spherical component. As a result, the couple-stress tensor is determinate in skew-symmetric C-CST. Interestingly, this shows that there is an interrelationship between the consistent mechanical boundary condition $(12), m^{(n n)}=0$, and the determinacy of the couple-stress tensor; resolving one, resolves the other (Hadjesfandiari and Dargush, 2011, 2015a,b).

For linear isotropic elastic materials, the constitutive relations in this theory are

$$
\begin{aligned}
\sigma_{(i j)} & =\lambda e_{k k} \delta_{i j}+2 \mu e_{i j} \\
\mu_{i j}= & -8 \eta \kappa_{i j} \\
& =-4 \eta\left(\omega_{i, j}-\omega_{j, i}\right)
\end{aligned}
$$

which shows that C-CST requires only one couple-stress material parameter.

As we can see, C-CST is a consistent extension of rigid body mechanics, which then is recovered in the absence of deformation. From a practical point of view, problems within C-CST are wellposed and boundary conditions can be satisfied precisely. For example, for the pure torsion of an elastic circular bar, the solution in C-CST reduces to that in classical theory, where there is no sizeeffect. Interestingly, this prediction completely agrees with recent experiments for pure torsion of micro-diameter copper wires ( $\mathrm{Lu}$ and Song, 2011; Song and Lu, 2015; Hadjesfandiari and Dargush, 2016b). Interestingly, in a work based on a discrete site-bond model, Morrison et al. (2013) have shown that a pure twist is not associated with any couple-stresses, which agrees with the C-CST prediction. In addition, C-CST describes the pure bending of a plate properly (Hadjesfandiari et al., 2016).

It is amazing to see that C-CST answers the criticism of Eringen about the indeterminacy of the couple-stress tensor in MTK-CST without adding any new artificial law. Contrary to the claim of Neff et al. (2016) and Sidhardh and Ray (2018), the skew symmetric character of the couple-stress tensor is not a postulate, but it has been proved systematically based on the developments of 
Mindlin and Tiersten (1962) and Koiter (1964) on the boundary conditions. Interestingly, the skew-symmetric character of the couple-stress tensor as a fundamental property can also be established as the result of arguments from tensor analysis (Hadjesfandiari, 2013a). It turns out that C-CST is also very desirable from a practical view, because it requires only one couple-stress material parameter $\eta$ for a linear isotropic elastic material. Hadjesfandiari et al. (2015) provides the theoretical background for any continuum, including both solids and fluids. Therefore, C-CST offers a fundamental basis for the development of size-dependent theories in many multi-physics disciplines that may govern the behavior of continua at the smallest scales. Hadjesfandiari (2013b, 2014) has developed size-dependent piezoelectricity and thermoelasticity. Remarkably, C-CST has recently demonstrated its self-consistency by resolving fundamental issues in the existing continuous defect theory, which has resulted in consistent continuous defect theory (Hadjesfandiari and Dargush, 2018c). In this theory, the dislocation density tensor is skewsymmetric and can be represented by a vector. Interestingly, this development also establishes the dualism between geometry and statics of consistent continuous defect theory based on C-CST.

A number of in-plane solutions for two-dimensional isotropic elastic materials in MTK-CST remain valid under C-CST, if one simply ignores the indeterminacies in the stresses and inconsistencies in boundary condition specifications (e.g., Mindlin, 1963; Muki and Sternberg, 1965; Sternberg and Muki, 1967; Atkinson and Leppington, 1977; Huang et al., 1997; Gourgiotis and Georgiadis, 2011; Itou, 2013a-c; Zisis et al., 2014; Karuriya and Bhandakkar, 2017; Baxevanakis et al., 2017a,b). However, this peculiar characteristic does not justify MTK-CST as a valid theory for solving all problems, such as torsion and anti-plane deformation. It is just serendipitous that certain aspects of solutions in MTK-CST for two-dimensional isotropic elastic problems are still useful in C-CST. Unfortunately, the inconsistent MTK-CST is still used for size-dependent problems (e.g., Itou, 2013a-c; Zisis et al., 2014; Karuriya and Bhandakkar, 2017; Baxevanakis et al., 2017a,b; Soldatos, 2018). Moreover, it is surprising to see that some authors still include the body couple in the formulation (e.g., Gourgiotis and Piccolroaz, 2014) or that in some dynamic formulations the effect of inertia is accounted by an extra micro-inertia in the analysis (e.g., Mishuris et al., 2012; Gourgiotis and Piccolroaz, 2014). However, the concept of moment of inertia per volume is meaningless in continuum mechanics. This is because the moment of inertia of a body depends on mass density and its distribution within the body. Defining the 
moment of inertia density does not make sense physically. The inertia of matter is accounted entirely by its mass density $\rho$ in consistent continuum mechanics.

\section{Discussion}

We have demonstrated that MTK-CST and M-CST are not consistent continuum mechanics theories for developing new size-dependent formulations, including those involving multi-physical responses. We notice that MTK-CST has a fundamental position in the evolution toward the consistent couple stress theory, whereas M-CST has no scientific value.

The main reasons for the popularity of M-CST are the appearance of a single couple-stress parameter $\eta$ in the constitutive relations for linear isotropic elastic material (e.g., Goncalves et al., 2017), and its ability to account for size-effects in beam bending. It cannot be denied that consistent theories are usually simple. This can be seen in the consistent couple stress theory (CCST), where the number of the couple-stress parameters for a linear isotropic elastic material is one. This clearly shows that C-CST is not only consistent, but also simple. Interestingly, for centrosymmetric cubic elastic materials, such as single crystal silicon, C-CST still requires only one couple-stress parameter, whereas M-CST requires two couple-stress parameters. This clearly demonstrates the superiority of C-CST over M-CST regarding the material properties, although the inherent self-consistency of C-CST is the main reason for accepting it as the valid theory.

In-plane solutions for two-dimensional deformation of isotropic elastic material remain valid in all couple stress theories (MTK-CST, M-CST and C-CST), if one simply ignores the indeterminacies in the stresses and inconsistencies in boundary condition specifications in MTK-CST and M-CST. This is the main reason for the apparent success of M-CST in describing size-effects for beam bending. However, we should notice that the out-of-plane solutions for these M-CST twodimensional problems are incorrect, because the couple-stresses in this theory create anticlastic deformation, not real bending. As demonstrated, M-CST is based on the torsion tensor $\chi_{i j}$, which is not a proper bending deformation. This is the reason why this apparently simple theory cannot represent the torsion of cylindrical bars and bending of plates properly (Hadjesfandiari et al., 
2016). Contrary to belief of the proponents of M-CST, the size-effect in this theory is the result of torsion or anticlastic deformation, rather than bending. Therefore, the three dimensional finite element development for M-CST implemented by Kwon and Lee (2017) cannot analyze real problems correctly.

It is really curious to see that Gao and Zhang (2016a,b) and Zhang et al. (2017) use the surface curvature tensor to include a surface effect, but use the torsion tensor $\chi_{i j}$ in the volume to include the bulk couple-stress effect in their developments. As a result, their formulations predict:

- no couple-stress size effect, but the greatest surface effect for pure spherical bending;

- most significant couple-stress size effect, but little or zero surface effect for pure anticlastic saddle bending.

However, even our mechanics intuition and physical reality require that the surface and couplestress effects should simultaneously appear to be greatest for pure spherical bending and the least for pure anticlastic saddle bending. This simply indicates that we should use the mean curvature tensor $\kappa_{i j}$ in the volume to account for the bulk couple-stress effect, not the torsion tensor $\chi_{i j}$. The couple-stresses in C-CST create synclastic or ellipsoidal cap-like deformation for surface elements of the continuum. As a result, C-CST always describes the size-effect in beam, plate and shell bending correctly. It is amazing to see that C-CST answers the criticism of Eringen about the indeterminacy of the couple-stress tensor in MTK-CST without adding any new artificial law. This clearly is the character of a consistent theory.

Interestingly, Tang (1983) has applied MTK-CST based beam bending of Kao et al. (1979) and Tzung et al. (1981) to examine four-point bending and uniaxial tensile data of various size cylindrical and square specimens for three grades of graphite: H-327, H-451, and AGOT. The evaluations indicate that the data can be interpreted by linear couple stress theory (MTK-CST) with $\frac{\eta^{\prime}}{\eta} \approx-0.85$ for H-451 graphite. Furthermore, the results are improved by considering a non- 
linear effect, which yields the new value $\frac{\eta^{\prime}}{\eta} \approx-0.96$. As we can see, these results in hindsight show that the experimental data actually approach the consistent couple stress theory (C-CST) corresponding to $\frac{\eta^{\prime}}{\eta}=-1$. On the other hand, one would need $\frac{\eta^{\prime}}{\eta} \rightarrow+1$ for verification of MCST, which clearly is not consistent with this experimental data for graphite.

We also notice that modified couple stress theory (M-CST) is not suitable to describe some multiphysics phenomena, such as size-dependent piezoelectricity. Interestingly, some experiments have reported about this size-effect phenomena in centrosymmetric materials. Therefore, in general size-dependent piezoelectricity, the piezoelectric effect can be generated in all dielectrics even in isotropic and centrosymmetric dielectric materials (Tagantsev, 1986; Eliseev et al., 2009; Hadjesfandiari, 2013b). However, an M-CST based size-dependent piezoelectricity cannot account for the flexoelectricity in isotropic and centrosymmetric materials. In this theory, the piezoelectric and flexoelectric tensors, which couple the symmetric measures of deformation $e_{i j}$ and $\chi_{i j}$ to the electric field $E_{i}$, have the same symmetry characters. As a result, both piezoelectric and flexoelectric tensors vanish simultaneously for centrosymmetric materials. We can see this inconsistency is the direct result of the fact that the symmetric curvature tensor $\chi_{i j}$ is in reality a torsion tensor, which does not couple to the electric field $E_{i}$. Therefore, the results from the MCST based size-dependent piezoelectricity contradicts experiments for centrosymmetric materials. It turns out that the flexoelectricity effect is the result of the coupling between the electric field $E_{i}$ and the proper mean curvature tensor $\kappa_{i j}$, not the torsion tensor $\chi_{i j}$ (Hadjesfandiari, 2013b). Therefore, the flexoelectric effect can exist for centrosymmetric materials in C-CST, which agrees with experiments. Interestingly, for isotropic and centrosymmetric cubic dielectric materials, $\mathrm{C}$ CST requires just one flexoelectric coefficient.

Inconsistency of MTK-CST and M-CST can also be seen in deriving the governing equations for elastic bodies by an energy method to minimize the total potential energy. It is expected that a consistent couple stress elasticity satisfies the divergence free compatibility constraint $\omega_{i, i}=0$ in 
this minimization process automatically. In their variational formulations for couple stress theory, Park and Gao (2008), and Neff and his colleagues (Neff et al., 2016; Ghiba et al., 2016; Münch et al., 2015) derived the final governing equations by direct minimization of the total potential energy with this perception. As a result, their direct variational method apparently results in a trace free or deviatoric couple-stress tensor, which is peculiar. However, Hadjesfandiari and Dargush (2016a) have demonstrated that the direct minimization of the total potential energy for MTK-CST and M-CST violates the divergence free compatibility constraint $\omega_{i, i}=0$. Therefore, it is necessary to impose the constraint $\omega_{i, i}=0$ by using the Lagrange multiplier method. Interestingly, for MTK-CST and M-CST, the Lagrange multiplier is precisely the indeterminate spherical part of the couple-stress tensor. This means that the variational method results in a spherical component for the couple-stress tensor in MTK-CST and M-CST. Therefore, the couple-stress tensor does not become trace free as Park and Gao (2008) and Neff et al. (2016), in their misleadingly titled paper, have concluded from an incorrect direct unconstrained minimization of the total potential energy (Hadjesfandiari and Dargush, 2016a). How can the couple-stress tensor be trace free in MCST, when the unconstrained minimization of the total energy potential violates the divergence free compatibility constraint $\omega_{i, i}=0$ ? As explained before, a couple stress theory (CST) with a deviatoric symmetric couple-stress tensor, $\mu_{i i}=0$, is inconsistent and cannot be accepted on physical grounds.

There has also been some desperate efforts to define C-CST as a special case of the original MTKCST (Neff et al., 2016). However, C-CST is not a special case of the original MTK-CST in a physical sense, because a consistent theory should never be classified as a special case of an inconsistent theory. We also notice that C-CST uses a different curvature tensor from the original MTK-CST. It apparently seems that for isotropic linear elastic materials the formulation in MCST and C-CST can be obtained by letting $\eta^{\prime}=\eta$ and $\eta^{\prime}=-\eta$, respectively, in the original MTKCST. However, we must notice that these cases are excluded by the condition $-\eta<\eta^{\prime}<\eta$ for the indeterminate MTK-CST. In addition, this peculiarity is only valid for isotropic material. There is no such a simple analogy for general anisotropic material or nonlinear cases. 
To perceive better the status of MTK-CST, M-CST and C-CST in continuum mechanics, we can use a simple illustrative analogy to classical continuum mechanics (Hadjesfandiari and Dargush, 2016a). By taking MTK-CST as the general couple stress theory and considering M-CST and CCST as apparent special cases, we have the following analogy:

1. Inconsistent MTK-CST with $k_{i j}=\omega_{j, i}$ as the curvature tensor is analogous to an incorrect classical elasticity theory with $u_{i, j}$ as the strain tensor;

2. Inconsistent M-CST with $\chi_{i j}$ as the curvature tensor is analogous to an incorrect classical elasticity theory with $\omega_{i j}$ as the strain tensor;

3. Consistent C-CST with $\kappa_{i j}$ as the curvature tensor is analogous to the correct classical elasticity theory with $e_{i j}$ as the strain tensor.

Consistent physical theories can only be considered as a special case of a more general consistent physical theory. However, we should remember that MTK-CST stands as a fundamental pillar in the development of the consistent couple stress theory (C-CST). This is obvious from the fact that elements of C-CST are based on the original MTK-CST. It has been very unfortunate that Mindlin, Tiersten and Koiter missed the skew-symmetric character of the couple-stress tensor, although they correctly stated the character of the boundary conditions in couple stress theory. As a result, their very important, but non-physical, indeterminate couple stress theory (MTK-CST) with $k_{i j}=\omega_{j, i}$ as the curvature tensor is analogous to non-physical classical theory with $u_{i, j}$ as the strain tensor.

We have shown that the consistency of boundary conditions has played an important role in developing C-CST. It should be mentioned that the consistent boundary conditions are also of crucial importance in transforming boundary value problems into integral equation representations. Therefore, C-CST with the skew-symmetric couple-stress tensor is the continuum mechanics formulation suitable for developing different size-dependent solid, fluid and multiphysics problems. Hadjesfandiari (2013b, 2014) and Hadjesfandiari et al. (2015) have already 
developed consistent size-dependent piezoelectricity and thermoelasticity and fluid mechanics. The boundary integral formulations and boundary element developments based on C-CST clearly demonstrate the consistency and practicality of this theory (Hadjesfandiari and Dargush, 2012; Hadjesfandiari et al., 2013; Hajesfandiari et al., 2016, 2017; Mikulich et al., 2018).

\section{Conclusions}

In this paper, we have examined some theoretical and practical aspects of the three primary couple stress theories, namely, MTK-CST, M-CST and C-CST, based on the criticism of Eringen (1968) about the inconsistent indeterminacy of the couple-stress tensor in MTK-CST. We have noted that without answering the criticism of Eringen, Yang et al. (2002) have violated the foundations of mechanics by adding a new non-physical law to reduce the number of couple-stress material parameters for linear elastic isotropic materials to one. However, M-CST with a symmetric couple-stress tensor not only inherits all inconsistences from the original MTK-CST, but also suffers from additional troubles. Contrary to the claim of Yang et al. (2002), Münch et al., 2015 and Surana et al. (2017), the couple-stress tensor cannot be chosen symmetric, because it violates many mathematical and physical laws.

We should realize that when a theory is indeterminate, it means something is wrong or missed. When there is confusion in a scientific field, an inconsistent but apparently simple solution might become very popular, which in reality escalates the confusion in the field further. It is interesting to see that the original couple stress theory (MTK-CST) and modified couple stress theory (MCST) serve as a prime example of this phenomenon. Based on the investigations presented in this paper, we conclude that the indeterminate modified couple stress theory (M-CST) can be classified as a pseudo-science.

On the other hand, we have shown that consistent couple stress theory (C-CST) answers the criticism of Eringen (1968) about the indeterminacy of the couple-stress tensor in MTK-CST and provides a consistent and complete continuum mechanics. It is remarkable to see that C-CST systematically links efforts of the Cosserats, Mindlin, Tiersten and Koiter and others over the span of a century. 


\section{References}

Atkinson, C., Leppington, F.G., 1977. The effect of couple stresses on the tip of a crack. Int. J. Solids Struct. 13, 1103-1122.

Asghari, M., Kahrobaiyan, M.H., Ahmadian, M.T., 2010. A nonlinear Timoshenko beam formulation based on the modified couple stress theory. Int. J. Eng. Sci. 48(12), 1749-1761.

Asghari, M., Rahaeifard, M., Kahrobaiyan, M.H., Ahmadian, M.T., 2011. The modified couple stress functionally graded Timoshenko beam formulation. Mater. Des. 32(3), 1435-1443.

Baxevanakis, K.P., Gourgiotis, P.A. and Georgiadis, H.G., 2017a. Interaction of cracks with dislocations in couple-stress elasticity. Part I: Opening mode. Int. J. Solids Struct. 118-119, 179-191.

Baxevanakis, K.P., Gourgiotis, P.A. and Georgiadis, H.G., 2017b. Interaction of cracks with dislocations in couple-stress elasticity. Part II: Shear modes. Int. J. Solids Struct. 118-119, 192-203.

Chen, X., Meguid, S.A., 2015. Snap-through buckling of initially curved microbeam subject to an electrostatic force. Proc. R. Soc. A 471 (2177), 20150072.

Cosserat, E., Cosserat, F., 1909. Théorie des corps déformables (Theory of deformable bodies). A. Hermann et Fils, Paris.

deWit, R., 1970. Linear theory of static disclinations. J. Res. Nat. Bur. Stand. A. Phys. Chem. 77A (1), 651-680.

Eliseev, E.A., Morozovska, A.N., Glinchuk, M.D., Blinc, R., 2009. Spontaneous flexoelectric/flexomagnetic effect in nanoferroics. Phys. Rev. B. 79, 165433.

Ellis, R.W, Smith, C.W., 1967. A thin plate analysis and experimental evaluation of couple stress effects. Exp. Mech. 7, 372-380.

Eringen, A.C., 1968. Theory of micropolar elasticity, Fracture, vol 2, ed. H. Liebowitz, Academic Press, New York, 662-729.

Gao, X.L., 2015. A new Timoshenko beam model incorporating microstructure and surface energy effects. Acta Mech. 226 (2), 457-474.

Gao, X.L., Zhang, G.Y. 2016a. A non-classical Kirchhoff plate model incorporating microstructure, surface energy and foundation effects. Continuum Mech. Thermodyn. 28, 195213.

Gao, X.L., Zhang, G.Y. 2016b. A non-classical Mindlin plate model incorporating microstructure, surface energy and foundation effects. Proc. R. Soc. A 472 (2191), 20160275. 
Gavardinas, I.D., Giannakopoulos, A.E., Zisis, Th., 2017. A von Karman plate analogue for solving anti-plane problems in couple stress and dipolar gradient elasticity. Int. J. Solids Struct. 148-149, 169-180.

Ghiba, I-D., Neff, P., Madeo, A., Münch, I., 2016. A variant of the linear isotropic indeterminate couple-stress model with symmetric local force-stress, symmetric nonlocal force-stress, symmetric couple-stresses and orthogonal boundary conditions. Math. Mech. Solids, DOI: 1081286515625535.

Goncalves, B.R., Romanoff, J., 2018. Size-dependent modelling of elastic sandwich beams with prismatic cores. Int. J. Solids Struct. 136-137, 28-37.

Goncalves, B.R., Karttunen, A., Romanoff, J., Reddy, J.N., 2017. Buckling and free vibration of shear-flexible sandwich beams using a couple-stress-based finite element. Compos. Struct. 165, 233-241.

Gourgiotis, P.A. and Piccolroaz, A., 2014. Steady-state propagation of a mode II crack in couple stress elasticity. Int. J. Fract. 188(2), 119-145.

Gourgiotis, P.A., Georgiadis, H.G., 2011. The problem of sharp notch in couple-stress elasticity. Int. J. Solids Struct. 48(19), 2630-2641.

Hadjesfandiari, A.R., 2013a. On the skew-symmetric character of the couple-stress tensor. Preprint arXiv: 1303.3569.

Hadjesfandiari, A.R., 2013b. Size-dependent piezoelectricity. Int. J. Solids Struct. 50(18), 27812791.

Hadjesfandiari A.R. 2014. Size-dependent thermoelasticity. Lat. Am. J. Solids Stru. 2014, 11(9), 1679-1708.

Hadjesfandiari, A. R., Dargush, G.F., 2011. Couple stress theory for solids. Int. J. Solids Struct. 48 (18), 2496-2510.

Hadjesfandiari, A.R., Dargush. G.F., 2012. Boundary element formulation for plane problems in couple stress elasticity. Int. J. Numer. Meth. Eng. 89(5), 618-636.

Hadjesfandiari, A. R., Dargush, G.F., Hajesfandiari, A., 2013. Consistent skew-symmetric couple stress theory for size-dependent creeping flow. J. Non-Newton. Fluid. Mech. 196, 83-94.

Hadjesfandiari, A.R., Hajesfandiari, A., Dargush, G.F., 2015. Skew-symmetric couple-stress fluid mechanics. Acta Mech. 226 (3), 871-895.

Hadjesfandiari, A.R., Dargush, G.F., 2015a. Evolution of generalized couple-stress continuum theories: a critical analysis. Preprint arXiv: 1501.03112. 
Hadjesfandiari, A.R., Dargush, G.F., 2015b. Foundations of consistent couple stress theory. Preprint arXiv: 1509.06299.

Hadjesfandiari, A.R., Dargush, G.F., 2016a. Couple stress theories: Theoretical underpinnings and practical aspects from a new energy perspective. Preprint arXiv: 1611.10249.

Hadjesfandiari, A.R., Dargush, G.F., 2016b. Comparison of theoretical elastic couple stress predictions with physical experiments for pure torsion. Preprint arXiv: 1605.02556.

Hadjesfandiari, A.R., Hajesfandiari, A., Dargush, G.F., 2016. Pure plate bending in couple stress theories. Preprint arXiv: 1606.02954.

Hadjesfandiari, A.R., Dargush, G.F., 2018a. Fundamental governing equations of motion in consistent continuum mechanics. Preprint arXiv: 1810.04514.

Hadjesfandiari, A.R., Dargush, G.F., 2018b. An assessment of higher gradient theories from a continuum mechanics perspective. Preprint arXiv: 1810.06977.

Hadjesfandiari, A.R., Dargush, G.F., 2018c. Consistent continuous defect theory. Preprint arXiv: 1808.10744.

Hajesfandiari, A., Hadjesfandiari, A.R. and Dargush, G.F., 2016. Boundary element formulation for plane problems in size-dependent piezoelectricity. Int. J. Numer. Meth. Eng. 108(7), 667-694.

Hajesfandiari, A., Hadjesfandiari, A.R. and Dargush, G.F., 2017. Boundary element formulation for steady state plane problems in size-dependent thermoelasticity. Eng. Anal. Bound. Elem. 82, 210-226.

Hayat, T., Aziz, A., Muhammad, T. and Ahmad, B., 2015. Influence of magnetic field in threedimensional flow of couple stress nanofluid over a nonlinearly stretching surface with convective condition. PloS one, 10(12), e0145332.

Hayat, T., Muhammad, T., Alsaedi, A., 2017. On three-dimensional flow of couple stress fluid with Cattaneo-Christov heat flux. Chinese Journal of Physics, 55, 930-938.

Hoffman, O., 1964. On Bending of Thin Elastic Plates in the Presence of Couple Stress. J. Appl. Mech. 31(4), 706-707.

Huang, Y., Zhang, L., Guo, T.F., Hwang, K.C., 1997. Mixed mode near tip fields for cracks in materials with strain-gradient effects. J. Mech. Phys. Solids 45(3), 439-465.

Itou, S., 2013a. Effect of couple-stresses on the Mode I dynamic stress intensity factors for two equal collinear cracks in an infinite elastic medium during passage of time-harmonic stress waves. Int. J. Solids Struct. 50(10), 1597-1604.

Itou, S., 2013b. Effect of couple-stresses on the stress intensity factors for a crack in an infinite elastic strip under tension. Eur. J. Mech. A-Solid. 42, 335-343. 
Itou, S., 2013c. Effect of couple-stresses on the transient dynamic stress intensity factors for a crack in an infinite elastic medium under an impact stress wave. Int. J. Fract. 183(1), 99-104.

Kao, B.G., Tzung, F.K., Ho, H.F., 1979. Influences of the couple-stresses on the pure-bending of a circular cylinder. General Atomic Rep. GA-A15141.

Karttunen, A.T., Romanoff, J., Reddy, J.N., 2016. Exact microstructure-dependent Timoshenko beam element. Int. J. Mech. Sci. 111, 35-42.

Karuriya, A.N. and Bhandakkar, T.K., 2017. Plane strain indentation on finite thickness bonded layer in couple stress elasticity. Int. J. Solids Struct. 108, 275-288.

Koiter, W.T., 1964. Couple stresses in the theory of elasticity, I and II. Proc. Ned. Akad. Wet. (B) 67, 17-44.

Kong, S., Zhou, S., Nie, Z., Wang, K., 2008. The size-dependent natural frequency of BernoulliEuler micro-beams. Int. J. Eng. Sci. 46(5), 427-437.

Kwon, Y.R. and Lee, B.C., 2018. Three dimensional elements with Lagrange multipliers for the modified couple stress theory. Comput. Mech. 62(1), 97-110.

Laouadi, B., Lahmar, M., Bou-Saïd, B., Mouassa, A. and Boucherit, H., 2018. Analysis of couplestress effects in gas foil bearings using the VK Stokes micro-continuum theory. Lubrication Science. https://doi.org/10.1002/1s.1430

Layek G.C., Pati, N. C., 2018. Chaotic thermal convection of couple-stress fluid layer. Nonlinear Dyn. 91(2), 837-852.

Lazopoulos, K.A., 2009. On bending of strain gradient elastic micro-plates. Mech. Res. Commun. 36, 777-783.

Li, Y., Meguid, S.A., Fu, Y., Xu, D., 2014. Nonlinear analysis of thermally and electrically actuated functionally graded material microbeam. Proc. R. Soc. A 470 (2162), 20130473.

Lu, W.-Y., Song, B., 2011. Quasi-static torsion characterization of micro-diameter copper wires. Exp. Mech. 51, 729-737.

Ma, H.M., Gao, X.-L., Reddy, J.N., 2008. A microstructure-dependent Timoshenko beam model based on a modified couple stress theory. J. Mech. Phys. Solids 56(12), 3379-3391.

Mahabaleshwar, U.S., Sarris, I.E., Hill, A.A., Lorenzini, G., Pop, I., 2017. An MHD couple stress fluid due to a perforated sheet undergoing linear stretching with heat transfer. Int. J. Heat Mass Transfer, 105, 157-167.

Mikulich, O., Pasternak, I. and Andriichuk, O., 2018. Modification of boundary integral equation method for investigation of dynamic stresses for couple stress elasticity. Mech. Res. Commun. 91, 107-111. 
Mindlin, R.D., 1965. Second gradient of strain and surface-tension in linear elasticity. Int. J. Solids Struct. 1(4), 417-438.

Mindlin, R.D., 1963. Influence of couple-stresses on stress concentrations. Exp. Mech. 3, 1-7.

Mindlin, R.D., Tiersten, H.F., 1962. Effects of couple-stresses in linear elasticity. Arch. Rational Mech. Anal. 11, 415-488.

Mindlin, R.D. and Eshel, N.N., 1968. On first strain-gradient theories in linear elasticity. Int. J. Solids Struct. 4(1), 109-124.

Mishuris, G., Piccolroaz, A. and Radi, E., 2012. Steady-state propagation of a Mode III crack in couple stress elastic materials. Int. J. Eng. Sci. 61, 112-128.

Morrison, C.N., Jivkov, A.P., Yates, J.R. 2013. Meso-scale features and couple stresses in fracture process zone. 13th International Conference on Fracture. Beijing, China. June 16-21.

Morini, L., Piccolroaz, A. and Mishuris, G., 2014. Remarks on the energy release rate for an antiplane moving crack in couple stress elasticity. Int. J. Solids Struct. 51 (18), 3087-3100.

Muki, R., Sternberg, E., 1965. The influence of couple-stresses on singular stress concentrations in elastic solids. Zeit. Ange. Math. Phys. 16, 611-648.

Münch, I., Neff, P., Madeo, A., Ghiba, I-D., 2015. The modified indeterminate couple stress model: Why Yang et al.'s arguments motivating a symmetric couple stress tensor contain a gap and why the couple stress tensor may be chosen symmetric nevertheless. Preprint arXiv: 1512.02053.

Nandal, R. and Mahajan, A., 2018. Penetrative convection in couple-stress fluid via internal heat source/sink with the boundary effects. J. Non-Newton. Fluid. Mech. 260, 133-141.

Neff, P., Münch, I., Ghiba, I-D., Madeo, A., 2016. On some fundamental misunderstandings in the indeterminate couple stress model. A comment on recent papers of A.R. Hadjesfandiari and G.F. Dargush. Int. J. Solids Struct. 81, 233-243.

Neff, P., Ghiba, I-D., Madeo, A., Münch, I, 2015. Correct traction boundary conditions in the indeterminate couple stress model. Preprint arXiv: 1504.00448.

Park, S.K., Gao, X.-L., 2006. Bernoulli-Euler beam model based on a modified couple stress theory. J. Micromech. Microeng. 16(11): 2355-9.

Park, S. K., Gao, X.-L., 2008. Variational formulation of a modified couple stress theory and its application to a simple shear problem. Z. Angew. Math. Phys. 59, 904-917.

Radi, E., 2008. On the effects of characteristic lengths in bending and torsion on Mode III crack in couple stress elasticity. Int. J. Solids Struct. 45(10), 3033-3058. 
Reddy, J.N., 2011. Microstructure-dependent couple stress theories of functionally graded beams. J. Mech. Phys. Solids 59 (11), 2382-2399.

Shankar, B.M., Kumar, J. and Shivakumara, I.S., 2014. Stability of natural convection in a vertical couple stress fluid layer. Int. J. Heat Mass Transfer, 78, 447-459.

Shankar, B.M., Kumar, J. and Shivakumara, I.S., 2016. Stability of natural convection in a vertical dielectric couple stress fluid layer in the presence of a horizontal ac electric field. Appl. Math. Model. 40(9), 5462-5481.

Shivakumara, I.S. and Kumar, S.N., 2014. Linear and weakly nonlinear triple diffusive convection in a couple stress fluid layer. Int. J. Heat Mass Transfer, 68, 542-553.

Sidhardh, S., Ray, M. C., 2018. Element-free Galerkin model of nano-beams considering strain gradient elasticity. Acta Mech. https://doi.org/10.1007/s00707-018-2139-x.

Soldatos, K.P., 2018. On the characterisation of polar fibrous composites when fibres resist bending. Int. J. Solids Struct. https://doi.org/10.1016/j.ijsolstr.2017.10.001

Song, B., Lu, W.-Y., 2015. An improved experimental technique to characterize micro-diameter copper wires in torsion. Exp. Mech. 55, 999-1004.

Srinivasacharya, D. and Rao, G.M., 2016. Computational analysis of magnetic effects on pulsatile flow of couple stress fluid through a bifurcated artery. Comput Methods Programs Biomed. 137, 269-279.

Sternberg, E., Muki, R., 1967. The effect of couple-stresses on the stress concentration around a crack. Int. J. Solids Struct. 3, 69-95.

Stokes, V.K., 1984. Theories of fluids with microstructure, Springer, Berlin Heidelberg.

Stokes, V.K., 1971. Effects of couple stresses in fluids on the creeping flow past a sphere. Phys. Fluids 14, 1580-1582.

Stokes, V.K., 1966. Couple stresses in fluids. Phys. Fluids 9, 1709-1715.

Surana, K.S., Reddy, J.N., Nunez, D., Powell, M., 2015. A polar continuum theory for solid continua. International Journal of Engineering Research and Industrial Applications, 8 (2), 77-106.

Sunil, Devi, R., Mahajan, A., 2011. Global stability for thermal convection in a couple-stress fluid. Int. Commun. Heat. Mass Transf. 38(7), 938-942.

Sunil, Choudhary, S., Mahajan, A., 2013. Conditional stability for thermal convection in a rotating couple-stress fluid saturating a porous medium with temperature and pressure dependent viscosity. J. Geophys. Eng., 10(4), 045013.

Surana, K.S., Reddy, J.N., Powell, M., 2015. A polar continuum theory for fluent continua. International Journal of Engineering Research and Industrial Applications, 8 (2), 107-146. 
Surana, K.S., Joy, A.D., Reddy, J.N., 2017. Non-classical continuum theory for fluids incorporating internal and Cosserat rotation rates. Continuum Mechanics and Thermodynamics, 29(6), 1249-1289.

Tagantsev, A.K., 1986. Piezoelectricity and flexoelectricity in crystalline dielectrics. Phys. Rev. B. 34, 5883-5889.

Tang, P.Y., 1983. Interpretation of bend strength increase of graphite by the couple-stress theory. Comput. Struct. 16(1), 45-49.

Tripathi, D., Yadav, A. and Bég, O.A., 2017. Electro-osmotic flow of couple stress fluids in a micro-channel propagated by peristalsis. Eur. Phys. J. Plus. 132(4), 173.

Turkyilmazoglu, M., 2014. Exact solutions for two-dimensional laminar flow over a continuously stretching or shrinking sheet in an electrically conducting quiescent couple stress fluid. Int. J. Heat Mass Transfer, 72, 1-8.

Tzung, F.K., Kao, B.G., Ho, H.F., Tang, P-Y, 1981. The effect of couple stresses on the pure bending of a prismatic bar. 6th Int. Conf. on Struct. Mech. Reactor Tech. (SMiRT), Paris, France, 17-21 August 1981.

Voigt, W., 1887. Theoretische studien fiber die elastizitatsverhiltnisse der kristalle (Theoretical studies on the elasticity relationships of crystals). Abh. Gesch. Wissenschaften, 34.

Yang, F., Chong, A.C.M., Lam, D.C.C., Tong P., 2002. Couple stress based strain gradient theory for elasticity. Int. J. Solids Struct. 39(10), 2731-2743.

Zhang, G.Y., Gao, X.L. and Tang, S., 2017. A non-classical model for circular Mindlin plates incorporating microstructure and surface energy effects. Procedia IUTAM, 21, 48-55.

Zisis, Th., 2018. Anti-plane loading of microstructured materials in the context of couple stress theory of elasticity: half-planes and layers. Arch. Appl. Mech. 88(1-2), 97-110.

Zisis, Th., Gourgiotis, P.A., Baxevanakis, K.P. and Georgiadis, H.G., 2014. Some basic contact problems in couple stress elasticity. Int. J. Solids Struct. 51(11), 2084-2095. 